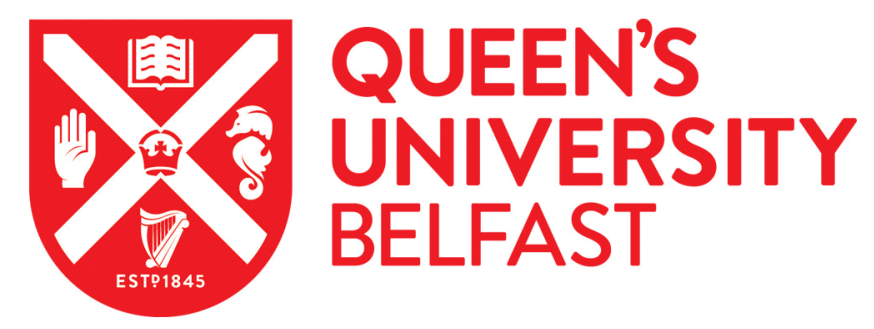

\title{
Relative subgroups in Chevalley groups
}

Hazrat, R. (2010). Relative subgroups in Chevalley groups. Journal of K-Theory, 5(3), 603-618.

\author{
Published in: \\ Journal of K-Theory
}

Queen's University Belfast - Research Portal:

Link to publication record in Queen's University Belfast Research Portal

\section{General rights}

Copyright for the publications made accessible via the Queen's University Belfast Research Portal is retained by the author(s) and / or other copyright owners and it is a condition of accessing these publications that users recognise and abide by the legal requirements associated with these rights.

Take down policy

The Research Portal is Queen's institutional repository that provides access to Queen's research output. Every effort has been made to ensure that content in the Research Portal does not infringe any person's rights, or applicable UK laws. If you discover content in the Research Portal that you believe breaches copyright or violates any law, please contact openaccess@qub.ac.uk. 


\title{
RELATIVE SUBGROUPS IN CHEVALLEY GROUPS
}

\author{
R. HAZRAT, V. PETROV, AND N. VAVILOV
}

\begin{abstract}
We finish the proof of the main structure theorems for a Chevalley group $G(\Phi, R)$ of rank $\geq 2$ over an arbitrary commutative ring $R$. Namely, we prove that for any admissible pair $(A, B)$ in the sense of Abe, the corresponding relative elementary group $E(\Phi, R, A, B)$ and the full congruence subgroup $C(\Phi, R, A, B)$ are normal in $G(\Phi, R)$ itself, and not just normalised by the elementary group $E(\Phi, R)$ and that $[E(\Phi, R), C(\Phi, R, A, B)]=E(\Phi, R, A, B)$. For the case $\Phi=\mathrm{F}_{4}$ these results are new. The proof is new also for other cases, since we explicitly define $C(\Phi, R, A, B)$ by congruences in the adjoint representation of $G(\Phi, R)$ and give several equivalent characterisations of that group and use these characterisations in our proof.
\end{abstract}

\section{INTRODUCTION}

In the present paper, which is a sequel of [7], we solve Problem 1 stated there and thus put the last dot in the proof of the main structure theorems for Chevalley groups of rank $\geq 2$ over commutative rings.

Namely, let $\Phi$ be a irreducible root system of rank $\geq 2$ and $R$ be a commutative ring. We consider the simply connected Chevalley group $G=G(\Phi, R)$ and its elementary subgroup $E(\Phi, R)$ generated by the unipotent root elements $x_{\alpha}(\xi), \alpha \in \Phi, \xi \in R$. Further, let $(A, B)$ be an admissible pair in the sense of Abe; in classical cases this means precisely that $B$ is a relative form parameter for $A$ in the sense of Bak. In $\S 2$ we recall the definition of the corresponding elementary subgroup $E(\Phi, R, A, B)$. For the time being we define the full congruence subgroup $C(\Phi, R, A, B)$ as the following transporter

$$
C(\Phi, R, A, B)=\{g \in G(\Phi, R) \mid[g, E(\Phi, R)] \leq E(\Phi, R, A, B)\},
$$

see [1]-[5] and [41].

Now we are in a position to state the first main result of the present paper.

Theorem 1. Let $\Phi$ be a reduced irreducible root system of rank $\geq 2, R$ be a commutative ring, $(A, B)$ an admissible pair. In the case, when $\Phi=\mathrm{B}_{2}$ or $\Phi=\mathrm{G}_{2}$ assume, moreover, that $R$ has no residue fields $\mathbb{F}_{2}$ of 2 elements. Then one has the following standard

2000 Mathematics Subject Classification. 20G15, 20 G35.

Key words and phrases. Chevalley groups, elementary subgroup, congruence subgroup, commutator formulas.

The first author acknowledges the support of EPSRC first grant scheme EP/D03695X/1. The work of the second author was financed by a PIMS postdoctoral fellowship at the University of Alberta. The work of the third author was financed through Hazrat's EPSRC first grant scheme at Queen's University Belfast. The second and the third authors were also supported by RFFI projects NSh-8464.2006.1 and 08-01-00756. 
commutator formulae:

$$
[G(\Phi, R), E(\Phi, R, A, B)]=[E(\Phi, R), C(\Phi, R, A, B)]=E(\Phi, R, A, B) .
$$

The extra assumption in the cases $\Phi=\mathrm{B}_{2}$ or $\Phi=\mathrm{G}_{2}$ is imposed only to guarantee that these are indeed equalities, and not just inclusions. The exceptional cases are exhaustively treated by Costa and Keller [15] and [16] and we do not dwell on them anymore.

In fact, this theorem seems to follow from the published results in most cases. Namely, for even unitary groups (including symplectic groups $=$ Chevalley groups of type $\mathrm{C}_{l}$ ) it is established in [10] and for odd unitary groups (including odd orthogonal groups = Chevalley groups of type $B_{l}$ ) - in [28]. For Chevalley groups of type $G_{2}$ it seems to follow from [16] (but will be proven again in the present paper). However, the most difficult case of Chevalley groups of type $\mathrm{F}_{4}$ seems to be new.

In fact, we do much more than that. We explicitly construct a subgroup $C(\Phi, R, A, B)$ that could be interpreted as the full congruence subgroup of level $(A, B)$ and prove the following result. The first of the equalities below is now an assertion, not a definition.

Theorem 2. Let $\Phi$ be a reduced irreducible root system of rank $\geq 2, R$ be a commutative ring, $(A, B)$ an admissible pair. Then the following four subgroups coincide:

$$
\begin{aligned}
C(\Phi, R, A, B) & =\{g \in G(\Phi, R) \mid[g, E(\Phi, R)] \leq E(\Phi, R, A, B)\} \\
& =\{g \in G(\Phi, R) \mid[g, E(\Phi, R)] \leq C(\Phi, R, A, B)\} \\
& =\{g \in G(\Phi, R) \mid[g, G(\Phi, R)] \leq C(\Phi, R, A, B)\} .
\end{aligned}
$$

Observe that, $C(\Phi, R, A, B)$ on the right hand side of these formulae could be replaced by any subgroup between $C(\Phi, R, A, B)$ and $E(\Phi, R, A, B)$ normal in $G(\Phi, R)$. One such subgroup is the principal congruence subgroup $G(\Phi, R, A, B)$, but for the exceptional cases we are most interested in, it coincides with $C(\Phi, R, A, B)$.

Together with results by Abe and Abe-Suzuki [1] - [5], Taddei [39], Vaserstein [41], Costa-Keller [15], [16] and Vavilov-Nikolenko [46], these theorems finish the proof of the main structure theorems for Chevalley groups of rank $\geq 2$, over an arbitrary commutative ring $R$.

In [46] we outlined three different approaches to the proof of the standard commutator formulae in the Chevalley group of type $\mathrm{F}_{4}$.

- Localisation proof in the style of [38], [9], [19].

- Relativisation proof, based on a version of Stein's relativisation [32] with two parameters, as developed in [10], [28].

- Explicit definition of the congruence subgroup $G\left(\mathrm{~F}_{4}, R, A, B\right)$ by congruences on matrix entries in the 26-dimensional (or, rather, 27-dimensional) representation, in the style of [47].

However, we were not quite happy with any of these approaches, for the following reasons.

- To implement a localisation proof, one should first develop commutator calculus, proving analogues of all subsidiary lemmas of, say, [38] or [19]. Routine, but very tedious. 
- To carry through relativisation with two parameters, for Chevalley groups of type $\mathrm{F}_{4}$, one should first define cubic form parameters, and the corresponding groups $G\left(\mathrm{~F}_{4}, R, \Lambda\right)$. Occasionally, we do know how to do it [11], but already the definitions are so technical that they make any further use of the group $G\left(\mathrm{~F}_{4}, R, \Lambda\right)$ appalling.

- It is easy to see, that $C\left(\mathrm{~F}_{4}, R, A, B\right)$ is a subgroup of $G\left(\mathrm{~F}_{4}, R\right)$, but to prove it is a normal subgroup, requires standard, but awkward calculations. It was precisely to avoid such calculations in the (much easier) case of unitary groups, that we developed relativisation with two parameters in [10].

Thus, we had to rethink the whole problem from scratch. A standard argument, based on the Hall-Witt identity and the fact that $E(\Phi, R, A, B)$ is $E(\Phi, R)$-perfect, shows that to prove Theorem 1 it suffices to exhibit some normal subgroup in $G=G(\Phi, R)$ between $E(\Phi, R, A, B)$ and $C(\Phi, R, A, B)$. For the case of the general linear group this argument can be found in the paper [33] by Alexei Stepanov.

It remained only to observe that, in fact, congruence subgroups are defined not by ideals of $R$, but rather by submodules of $G$-modules. One such module is, of course, the adjoint module $L=L_{R}$. The ideals of $L_{R}$ were described by James Hurley [21] back in 1969, and they correspond to admissible pairs. Modulo elementary facts about $E(\Phi, R, A, B)$, this proof does not use anything beyond elementary linear algebra and group theory. It is conceptual, rather than computational and naturally accounts for several hitherto unrelated facts.

All necessary facts concerning Chevalley groups over rings can be found in [1] - [5], [26], [31], [42], [48].

\section{Main Structure theorems}

The main structure theorems are stated in terms of relative subgroups, which in the case of simply laced systems depend on one parameter, usually interpreted as an ideal $I \unlhd R$. Namely, one considers the reduction homomorphism $\rho_{I}: G(\Phi, R) \longrightarrow G(\Phi, R / I)$ and defines the following three relative subgroups of level $I$.

- The relative elementary subgroup $E(\Phi, R, I)$, defined as the normal subgroup of the absolute elementary group $E(\Phi, R)$, generated by all elementary root unipotents $x_{\alpha}(\xi)$, $\alpha \in \Phi, \xi \in I$, of level $I$.

- The principal congruence subgroup $G(\Phi, R, I)$ of level $I$, defined as the kernel of the reduction homomorphism $\rho_{I}$ modulo $I$.

- The full congruence subgroup $C(\Phi, R, I)$ of level $I$, defined as the inverse image of the centre of $G(\Phi, R / I)$ with respect to the reduction homomorphism $\rho_{I}$ modulo $I$.

It turns out, that for simply laced systems, and under some additional assumptions also for multiply laced systems, one has the following results, known as the main structure theorems. 
- For every subgroup $H \leq G(\Phi, R)$ normalised by $E(\Phi, R)$ there exists a unique ideal $I \unlhd R$ such that

$$
E(\Phi, R, I) \leq H \leq C(\Phi, R, I)
$$

- The following standard commutator formulae hold

$$
[G(\Phi, R), E(\Phi, R, I)]=E(\Phi, R, I)=[E(\Phi, R), C(\Phi, R, I)] .
$$

It is not feasible even to sketch the development of these results for the classical groups here, see [17], [42], [10], [20], [34], [8] for details and further references. Since in the present paper we are only concerned with the second of these results, let us mention, that for classical groups the first equality was proved by Andrei Suslin and Vyacheslav Kopeiko [36], [37], [23] and the second one by Leonid Vaserstein, Zenon Borewicz and the third author, and Li Fuan, see [40], [13], [24], [25] (and subsequent papers by Vaserstein, for more general related results).

For exceptional groups the first proofs of these results were based on localisation and were obtained and by Eiichi Abe [1] - [4], Abe and Kazuo Suzuki [5], and Vaserstein [41], and by Giovanni Taddei [39] and Vaserstein [41], respectively.

Subsequently, new proofs of the standard commutator formulae and their generalisations were published by many authors, see, for example, [42] [38], [9], [33], [10], [34], [18], [19], [35]. New proofs were published also for the description of normal subgroups [42], [34], [44], [46], [49], and further references in [45] and [43] $]^{1}$.

Recently two new contexts emerged, of remarkable generality, when the elementary subgroup has been shown to be normal. One of them is the work of the second author on odd unitary groups, [27], [28]. Another one is a very recent paper of the second author and Anastasia Stavrova [29], where normality of the elementary subgroup is established for the group of points of an isotropic reductive group over an arbitrary commutative ring, under the assumption that all localisations have only components of semi-simple ranks $\geq 2$ (see [29] for the precise statements).

However, a correct analogue of these results for multiply laced systems should be stated in more general terms. For classical groups this was discovered by Anthony Bak [6], see Hyman Bass [12] for an exposition, and for exceptional groups - by Eiichi Abe [1], the same year! Namely, for classical groups ideals should be replaced by form ideals $(I, \Gamma)$, see [6], [12], [17], [10], and in general — by admissible pairs $(A, B)$, see [1] - [5]. Almost simultaneously admissible pairs were introduced — in related, but somewhat different contexts! — by James Hurley, see in particular [21], [22], and Michael Stein [30], [31].

Let $A$ be an ideal of $R$. Denote by $A_{2}$ the ideal, generated by $2 \xi$ and $\xi^{2}$ for all $\xi \in A$. The first component $A$ of an admissible pair $(A, B)$ is an ideal of $R$, parametrising short roots. When $\Phi \neq C_{l}$ the second component $B, A_{2} \leq B \leq A$, is also an ideal, parametrising long roots. In the exceptional case $\Phi=C_{l}$ the second component $B$ is an additive subgroup

\footnotetext{
${ }^{1}$ Since we are only interested in Chevalley groups, here we do not mention remarkable non-commutative generalisations, published mostly by the Moscow School, Vaserstein himself, Alexander V. Mikhalev, Igor Golubchik and Sergei Khlebutin, and by others, including Anthony Bak, Alexei Stepanov, Hong You, and ourselves.
} 
stable under multiplication by $\xi^{2}, \xi \in R$ (in other words, it is a relative form parameter in the sense of Bak). A similar notion can be introduced for the type $G_{2}$ as well, but in this case one should replace 2 by 3 everywhere in the above definition.

Now, the relative elementary subgroup, corresponding to an admissible pair $(A, B)$, is defined as follows:

$$
E(\Phi, R, A, B)=\left\langle x_{\alpha}(\xi), \alpha \in \Phi_{s}, \xi \in A ; x_{\beta}(\zeta), \beta \in \Phi_{l}, \zeta \in B\right\rangle^{E(\Phi, R)} .
$$

where $\Phi_{s}$ and $\Phi_{l}$ are the sets of short and long roots in $\Phi$, respectively.

These notions take into account the fact that in general some coefficients in the Chevalley commutator formula are not invertible, and thus a normal subgroup may have two different levels, the upper level, defined in terms of short roots, and the lower level, defined in terms of long roots. Let $\delta$ and $\eta$ be the long and the short dominant roots of $\Phi$, respectively. Further, let $H \leq G(\Phi, R)$. Then the additive subgroups

$$
A=\left\{\xi \in R \mid x_{\eta}(\xi) \in H\right\}, \quad B=\left\{\xi \in R \mid x_{\delta}(\xi) \in H\right\}
$$

are called the upper and the lower level of $H$, respectively. Occasionally we will refer to the pair $(A, B)$ as the level of $H$.

In view of the standard description of normal subgroups, Lemma 4 below, for subgroups normalised by $E(\Phi, R)$ these notions happen to coincide with the classical notions of the lower and upper level, defined as the largest ideal such that $E(\Phi, R, B) \leq H$ and the smallest ideal such that $H \leq C(\Phi, R, A)$.

By the very definition the relative elementary subgroup $E(\Phi, R, A, B)$, is normal in the absolute elementary group $E(\Phi, R)$. We wish to prove that it is normal in $G(\Phi, R)$. Thereby we use the following results, which can be found in [31] or [3]. These facts are elementary in the sense, that they rely exclusively on the Chevalley commutator formula.

Lemma 1. Let $\operatorname{rk}(\Phi) \geq 2$. In the case, when $\Phi=\mathrm{B}_{2}$ or $\Phi=\mathrm{G}_{2}$ assume, moreover, that $R$ has no residue fields $\mathbb{F}_{2}$ of 2 elements. Then the elementary subgroup $E(\Phi, R, A, B)$ is $E(\Phi, R)$-perfect, in other words,

$$
[E(\Phi, R), E(\Phi, R, A, B)]=E(\Phi, R, A, B) .
$$

In particular, $E(\Phi, R)$ is perfect.

Lemma 2. As a subgroup $E(\Phi, R, A, B)$ is generated by the elements $x_{-\alpha}(\zeta) x_{\alpha}(\xi) x_{-\alpha}(-\zeta)$, where $\xi \in A$ for $\alpha \in \Phi_{s}$ and $\xi \in B$ for $\alpha \in \Phi_{l}$, while $\zeta \in R$.

Lemma 3. Let $\operatorname{rk}(\Phi) \geq 2$. Then the upper and lower levels of a subgroup $H \leq G(\Phi, R)$ normalised by $E(\Phi, R)$ form an admissible pair. This is the largest admissible pair $(A, B)$ such that $E(\Phi, R, A, B) \leq H$.

With the same definition of the group $C(\Phi, R, A, B)$ as in the introduction, Abe [2] [4] establishes the following result (which relies on the previous work by Abe - Suzuki [1], [5], Taddei [39] and Vaserstein [41]). Observe, that this fact is not elementary in the above sense, in that it relies on results, whose proof uses localisation. 
Lemma 4. Let $\operatorname{rk}(\Phi) \geq 2$. In the case, when $\Phi=\mathrm{B}_{2}$ or $\Phi=\mathrm{G}_{2}$ assume, moreover, that $R$ has no residue fields $\mathbb{F}_{2}$ of 2 elements. Then for every subgroup $H \leq G(\Phi, R)$ normalised by $E(\Phi, R)$ there exists a unique admissible pair $(A, B)$ such that

$$
E(\Phi, R, A, B) \leq H \leq C(\Phi, R, A, B) .
$$

The answer in the exceptional cases requires a further modification of the notion of level. Such a generalisation, known as radix, was introduced by Douglas Costa and Gordon Keller in their amazing papers [15] and [16], but its far too technical to be defined here.

However, this only generalises the first of the above structure theorems. To the best of our knowledge analogues of the second one, with ideals replaced by admissible pairs, can be found in the existing literature in most, but not in all cases. In particular, it is natural to ask the following questions.

- Is the relative elementary group $E(\Phi, R, A, B)$ normal not only in the elementary group $E(\Phi, R)$, but in the Chevalley group $G(\Phi, R)$ itself?

- Does the second commutator formula hold in this setting?

- Moreover, for one parameter the full congruence subgroup is defined as a different transporter, which in our case should look as

$$
C(\Phi, R, A, B)=\{g \in G(\Phi, R) \mid[g, G(\Phi, R)] \leq G(\Phi, R, A, B)\}
$$

or, what is the same, as

$$
C(\Phi, R, A, B)=\{g \in G(\Phi, R) \mid[g, E(\Phi, R)] \leq G(\Phi, R, A, B)\},
$$

where $G(\Phi, R, A, B)$ is the principal congruence subgroup of level $(A, B)$, which should be normal in $G(\Phi, R)$.

But in the existing literature one cannot even find the definition of $G(\Phi, R, A, B)$, see the discussion in [46], [49]. In the present paper we fill all these gaps by proving Theo-

rems 1 and 2 above. To prove them, we actually define the principal congruence subgroup $G(\Phi, R, A, B)$, thereby clarifying, what a congruence subgroup is in general.

\section{Congruence Subgroups}

First, let $G$ be any group acting on a right $R$-module $V$ and let $U \leq V$ be a $G$-submodule. Then we can define a set

$$
G(V, U)=\{g \in G \mid \forall v \in V, g v-v \in U\} .
$$

The following obvious observation is crucial for our purposes.

Lemma 5. For any $U \leq V$, the set $G(V, U)$ is a normal subgroup of $G$.

Proof. Let $g, h \in G(V, U)$ and $v \in V$. Then $g h v-v=(g h v-h v)+(h v-v) \in U$ and $g^{-1} v-v=g^{-1}(v-g v) \in g^{-1} U=U$. Finally, if $h \in G(V, U)$ and $g \in G$, then $g^{-1} h g v-v=g^{-1}(h g v-g v) \in g^{-1} U=U$. 
In the sequel we call $G(V, U)$, the congruence subgroup modulo $U$. By the very definition, the factor-group $G / G(V, U)$ acts on $V / U$. Since for every $g \notin G(V, U)$, there exists a $v \in V$ such that $g v \notin v+U$, this action is faithful. Let us list some obvious properties of this construction:

- If $U \leq W \leq V$, then $G(V, U) \leq G(U, W)$.

- Let $U_{1} \leq V_{1}$ and $U_{2} \leq V_{2}$. Then $G\left(V_{1} \oplus V_{2}, U_{1} \oplus U_{2}\right)=G\left(V_{1}, U_{1}\right) \cap G\left(V_{2}, U_{2}\right)$.

Now, we are all set to apply this construction to a Chevalley group $G(\Phi, R)$ and its rational module $V$. As usual, to distinguish the simply-connected Chevalley group and the adjoint group, we write $G_{\mathrm{sc}}(\Phi, R)$ and $G_{\mathrm{ad}}(\Phi, R)$. Further, let $P(\Phi)_{++}$be the cone of integral dominant weights. Our modules are Weyl modules $V=V(\omega)$, with the highest weight $\omega \in P(\Phi)_{++}$. It is well-known that $V$ is faithful as an algebraic representation if and only if $\omega$ generates $P / Q(\Phi)$, where $P$ is the lattice corresponding to $G$.

In our new terms the usual congruence subgroups are interpreted as follows. For any module $V$ and any ideal $I \unlhd R$ the product $U=V I$ is a $G$-submodule (actions of $G$ and $R$ commute). Here are the two archetypical examples.

Lemma 6. When $V$ is faithful, $G(V, V I)=G(\Phi, R, I)$ is the usual principal congruence subgroup of level $I$.

Proof. Follows from the commutative diagram

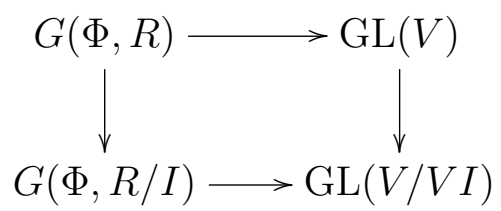

whose horizontal maps are injective by the assumption.

Lemma 7. When $V=L$ is the Lie algebra of $G(\Phi, R)$, considered as the adjoint module, then $G(L, L I)=C(\Phi, R, I)$ is the usual full congruence subgroup of level $I$.

Proof. Indeed, the action of $G(\Phi, R)$ on $L$ factors through the faithful action of the adjoint group $G_{\mathrm{ad}}(\Phi, R)$. Hence $C(\Phi, R, I)$ coincides with the inverse image of the congruence subgroup in $G_{\text {ad }}(\Phi, R)$.

However, it may happen - and it does happen! — that $V$ has $G$-submodules other, than those of the form $V I$. Since we are mostly interested in the case of exceptional groups, we consider the case when $V=L$ is the Lie algebra of $G$.

Fix an order of the root system $\Phi$, let $\Pi=\left\{\alpha_{1}, \ldots, \alpha_{l}\right\}$ be the corresponding set of fundamental roots. Choose in $L$ a Cartan subalgebra $H$ and let $e_{\alpha}, \alpha \in \Phi$ and $h_{\alpha_{i}}, \alpha_{i} \in \Pi$ be a Chevalley base of $L$. Here $h_{\alpha}=\left[e_{\alpha}, e_{-\alpha}\right]$ is a co-root, corresponding to the root $\alpha$. Further, let $N_{\alpha \beta}$ be the structure constants in this base, $\left[e_{\alpha}, e_{\beta}\right]=N_{\alpha \beta} e_{\alpha+\beta}$ and

$$
N_{\alpha \beta i 1}=\frac{1}{i !} N_{\alpha \beta} N_{\alpha, \beta+\alpha} \ldots N_{\alpha, \beta+(i-1) \alpha} .
$$


Finally, $A_{\beta \alpha}=2(\beta, \alpha) /(\beta, \beta)$ denotes Cartan numbers.

The action of elementary root unipotents in a Chevalley base is classically known, see for example [14]. We summarise it for subsequent use.

Lemma 8. The action of the elementary root unipotent $x_{\alpha}(\xi), \alpha \in \Phi, \xi \in R$, is described by the following formulae

$$
\begin{aligned}
& x_{\alpha}(\xi) e_{\alpha}=e_{\alpha}, \\
& x_{\alpha}(\xi) e_{-\alpha}=e_{-\alpha}+h_{\alpha} \xi-e_{\alpha} \xi^{2}, \\
& x_{\alpha}(\xi) e_{\beta}=e_{\beta}+\sum_{i=1}^{q} e_{\beta+i \alpha} N_{\alpha \beta i 1} \xi^{i}, \\
& x_{\alpha}(\xi) h_{\beta}=h_{\beta}+e_{\alpha} A_{\beta \alpha} \xi
\end{aligned}
$$

where $q$ is the largest integer such that $\beta+q \alpha \in \Phi$.

On the other hand, the element of the torus $T(\Phi, R)$ act on $L$ in an obvious way:

$$
t e_{\alpha}=e_{\alpha} \alpha(t), \quad t h_{\alpha}=h_{\alpha} .
$$

Now it is easy to construct $G(\Phi, R)$-submodules of the Lie algebra $L$ which do not have the form $L I$ for an ideal $I \unlhd R$. In fact, denote by $L_{l}$, or respectively by $L_{s}$, the span of all $e_{\alpha}$ and $h_{\alpha}$, where $\alpha$ is long, or respectively, short.

Let $(A, B)$ be an admissible pair. Consider the submodule $L_{l} B+L_{s} A$ in $L$. This is a Lie ideal in $L$ [21]. We prove that this is in fact a $G(\Phi, R)$-submodules.

Lemma 9. The module $U=L_{l} B+L_{s} A$ is $E(\Phi, R)$-invariant.

Proof. This follows from Lemma 8. Indeed, $x_{\alpha}(\xi)$ carries the element $e_{\alpha} \zeta \in U$ to an element of $U$ simply because $A$ and $B$ are ideals. Similarly, $x_{\alpha}(\xi)$ carries the element $h_{\beta} \zeta \in U$ to an element of $U$ because $A$ and $B$ are ideals if $|\alpha|=|\beta|$, because $B \leq A$ if $\alpha$ is short and $\beta$ is long, and because $p A \leq B$ if $\alpha$ is long and $\beta$ is short, where $p=2$ for $\Phi \neq \mathrm{G}_{2}$ and $p=3$ in this last case (compare, for example, [48], Table IV). It remains to verify, why $x_{\alpha}(\xi)$ carries the element $x_{\beta} \zeta \in U, \beta \neq-\alpha$, to an element of $U$. Considering all possible configurations of pairs of roots, we see that this again follows from the fact that $A$ and $B$ are ideals, $B \leq A$ and $3 A \leq B$ or, respectively $2 A \leq B$, depending on whether $\Phi \neq \mathrm{G}_{2}$ or not.

Lemma 10. The module $U=L_{l} B+L_{s} A$ is $G(\Phi, R)$-invariant.

Proof. We have to prove that $g u$ belongs to $L_{l} B+L_{s} A$ for all $g \in G(\Phi, R), u \in L_{s} A+L_{l} B$. By the local-global principle it suffices to show that in all localisations at maximal ideals. Since $\left(L_{l} B+L_{s} A\right)_{M}=L_{l M} B_{M}+L_{s M} A_{M}$, we may assume that $R$ is a local ring. But then $G(\Phi, R)=E(\Phi, R) T(\Phi, R)$, and the assertion follows from Lemma 9 and the fact, that $T$ acts diagonally on all generators of $U$, whereas $A$ and $B$ are ideals. 
RELATIVE SUBGROUPS IN CHEVALLEY GROUPS

Now we define $\tilde{C}(\Phi, R, A, B)$ as $G\left(L, L_{l} B+L_{s} A\right)$; by Lemma 5 it is a normal subgroup in $G(\Phi, R)$. In the next section we prove that $\tilde{C}(\Phi, R, A, B)=C(\Phi, R, A, B)$, where the latter is defined in the introduction.

\section{Standard commutator formulae}

In this section we prove Theorems 1 and 2. Throughout the section we assume, that $\Phi$ is a reduced irreducible root system of rank 2 , and, moreover, when $\Phi=\mathrm{B}_{2}$ or $\Phi=\mathrm{G}_{2}$ that the ring $R$ does not have the residue field $\mathbb{F}_{2}$ of 2 elements. Further, $(A, B)$ is an admissible pair in $R$.

The following result immediately follows from the Chevalley commutator formula. Its detailed verification can be found in [31], [3].

Lemma 11. The elementary subgroup $E(\Phi, R, A, B)$ is $E(\Phi, R)$-perfect, in other words,

$$
[E(\Phi, R), E(\Phi, R, A, B)]=E(\Phi, R, A, B) .
$$

We prove the next result for $\Phi \neq \mathrm{C}_{l}$ - for the symplectic group Theorems 1 and 2 are known anyway, see [10].

Lemma 12. Assume, that $\Phi \neq \mathrm{C}_{l}$. Then

$$
E(\Phi, R, A, B) \leq \tilde{C}(\Phi, R, A, B) \leq C(\Phi, R, A, B) .
$$

Proof. First, we prove that the level of $\tilde{C}(\Phi, R, A, B)$ equals $(A, B)$. Indeed, let $x_{\alpha}(\xi) \in$ $\tilde{C}(\Phi, R, A, B)$. If there is a root $\beta$ of the same length as $\alpha$ forming the angle $2 \pi / 3$ with it, then $x_{\alpha}(\xi) e_{\beta}-e_{\beta}= \pm e_{\beta+\alpha} \xi \in U$, where $U$ is as in Lemmas 9 and 10 , so that by condition $\xi \in A$ or $\xi \in B$, depending on whether $\alpha$ is short or long. This already accounts for all cases, except $\Phi=\mathrm{C}_{l}$ and $\alpha$ is long or $\Phi=\mathrm{B}_{l}, \mathrm{G}_{2}$ and $\alpha$ is short. When $\alpha$ is short, there exists a long root $\beta$ forming mutual angle $3 \pi / 4$ or $5 \pi / 6$ with it and then $x_{\alpha}(\xi) e_{\beta}-e_{\beta}= \pm e_{\beta+\alpha} \xi+\cdots \in U$. Since $\beta+\alpha$ is necessarily short, this again guarantees that $\xi \in A$ in this case.

Now, since $\tilde{C}(\Phi, R, A, B)$ is normal in $G(\Phi, R)$ by Lemma 5 and, thus in particular, $E(\Phi, R)$-normalised, the inequality $\tilde{C}(\Phi, R, A, B) \leq C(\Phi, R, A, B)$ follows from the Normal Structure Theorem, Lemma 4.

In fact, as we see below as part of the proof of Theorem 2, one has even $\tilde{C}(\Phi, R, A, B)=$ $C(\Phi, R, A, B)$. This is not needed to establish Theorem 1 , however.

Proof of Theorem 1. The inclusion

$$
[E(\Phi, R), C(\Phi, R, A, B)] \leq E(\Phi, R, A, B)
$$

is just the definition of the group $C(\Phi, R, A, B)$ given in the introduction. Next, we prove that $E(\Phi, R, A, B)$ is normal in $G(\Phi, R)$. In other words, we have to verify the inclusion

$$
[E(\Phi, R, A, B), G(\Phi, R)] \leq E(\Phi, R, A, B)
$$


In view of Lemma 11 , it suffices to show that $\left[[x, y],{ }^{y} z\right]$ belongs to $E(\Phi, R, A, B)$ for all $x \in E(\Phi, R, A, B), y \in E(\Phi, R), z \in G(\Phi, R)$. Recall the Hall-Witt commutator identity:

$$
\left[[x, y],{ }^{y} z\right]\left[[y, z],{ }^{z} x\right]\left[[z, x],{ }^{x} y\right]=1 .
$$

We show that the second and the third factors belong to $E(\Phi, R, A, B)$. Obviously, since $\tilde{C}(\Phi, R, A, B)$ is normal in $G(\Phi, R)$, the conjugates of matrices from $E(\Phi, R, A, B)$ fall into $\tilde{C}(\Phi, R, A, B)$. It follows that $[y, z] \in E(\Phi, R),{ }^{z} x \in \tilde{C}(\Phi, R, A, B)$, and thus the second factor is in $E(\Phi, R, A, B)$. Similarly, $[z, x] \in \tilde{C}(\Phi, R, A, B),{ }^{x} y \in E(\Phi, R)$, and thus the third factor is in $E(\Phi, R, A, B)$. This means that the first factor is in $E(\Phi, R, A, B)$ too, as claimed. These inclusions are equalities by Lemma 11.

Remark. There is another approach to the proof of Theorem 1, in the style of [2] - [4]. One could define $\tilde{C}(\Phi, R, A, B)$ as the group of all $g$ such that $F_{M}(g)$ (see below) belongs to $C\left(\Phi, R_{M}, A_{M}, B_{M}\right)$ for all maximal ideal $M$ in $R$ instead. Its normality follows from the fact that $G(\Phi, R)=E(\Phi, R) T(\Phi, R)$ when $R$ is local.

To prove Theorem 2, we have to invoke two further results by Eiichi Abe. The following lemma is [3, Sec. 4, Prop. I]. In the following lemma we denote by $F_{M}$ the localisation homomorphism $F_{M}: G(\Phi, R) \longrightarrow G\left(\Phi, R_{M}\right)$ at a maximal ideal $M$.

Lemma 13. For any $g \in G(\Phi, R)$, the inclusion $g \in C(\Phi, R, A, B)$ is equivalent to $F_{M}(g) \in$ $C\left(\Phi, R_{M}, A_{M}, B_{M}\right)$ for all maximal ideals $M \in \operatorname{Max}(R)$.

Further, denote

$$
T^{*}(\Phi, R, A, B)=T(\Phi, R) \cap C(\Phi, R, A, B) .
$$

The following result is established in [1] (see also [5] or [3, Lemma 4.4] for more general results in this direction).

Lemma 14. For any admissible pair $(A, B)$ in a local ring $R$ one has

$$
C(\Phi, R, A, B)=E(\Phi, R, A, B) T^{*}(\Phi, R, A, B) .
$$

We are now ready to prove Theorem 2.

Proof of Theorem 2. First, we show that $\tilde{C}(\Phi, R, A, B)=C(\Phi, R, A, B)$, so that, in particular, $C(\Phi, R, A, B)$ is normal in $G(\Phi, R)$. Let $g$ be an element of $C(\Phi, R, A, B)$; we have to show that $g v-v$ belongs to $L_{l} B+L_{s} A$ for all $v \in V$. By Lemma 13, we may assume that $R$ is local. But for local rings we can invoke Lemma 14. Now, $E(\Phi, R, A, B)$ is contained in $\tilde{C}(\Phi, R, A, B)$ by Lemma 12 . By the very definition, for any $t \in T^{*}(\Phi, R, A, B)$ and short, or respectively long root $\alpha$, we have $\alpha(t)$ is in $A$, or respectively, in $B$. Now the formulae for the action of $T(\Phi, R)$ shows that $T^{*}(\Phi, R, A, B)$ is contained in $\tilde{C}(\Phi, R, A, B)$ as well.

It only remains to prove the remaining two equalities. Denote

$$
\begin{aligned}
& C_{E, E}=\{g \in G(\Phi, R) \mid[g, E(\Phi, R)] \leq E(\Phi, R, A, B)\}, \\
& C_{E, G}=\{g \in G(\Phi, R) \mid[g, E(\Phi, R)] \leq C(\Phi, R, A, B)\}, \\
& C_{G, G}=\{g \in G(\Phi, R) \mid[g, G(\Phi, R)] \leq C(\Phi, R, A, B)\} .
\end{aligned}
$$


Obviously, $C_{E, E}, C_{G, G} \leq C_{E, G}$. On the other hand, $\left[C_{E, G}, E(\Phi, R)\right] \leq C(\Phi, R, A, B)$ and thus, the level of $C_{E, G}$ equals $(A, B)$ and $C_{E, G} \leq C(\Phi, R, A, B)=C_{E, E}$, by Lemma 4 . Finally, the inequality $C_{E, E} \leq C_{G, G}$ follows from the fact that $C(\Phi, R, A, B)=C_{E, E}$ is normal in $G(\Phi, R)$.

\section{REFERENCES}

[1] E. Abe, Chevalley groups over local rings, Tôhoku Math. J. 21 (1969), no.3, 474-494. 1, 2, 3, 4, 5, 10

[2] E. Abe, Chevalley groups over commutative rings, Proc. Conf. Radical Theory (Sendai, 1988), Uchida Rokakuho, Tokyo (1989), 1-23. 5, 10

[3] E. Abe, Normal subgroups of Chevalley groups over commutative rings, Algebraic $K$-Theory and Algebraic Number Theory (Honolulu, HI, 1987), Contemp. Math. 83 (1989), 1-17. 5, 9, 10

[4] E. Abe, Chevalley groups over commutative rings. Normal subgroups and automorphisms, Second International Conference on Algebra (Barnaul, 1991), Contemp. Math. 184 (1995), 13-23. 4, 5, 10

[5] E. Abe, K. Suzuki, On normal subgroups of Chevalley groups over commutative rings, Tôhoku Math. J. 28 (1976), no.1, 185-198. 1, 2, 3, 4, 5, 10

[6] A. Bak, The stable structure of quadratic modules, Thesis Columbia Univ., 1969. 4

[7] A. Bak, R. Hazrat, N. Vavilov, Localization-completion strikes again: relative $\mathrm{K}_{1}$ is nilpotent by abelian, J. of Pure and Appl. Algebra 213 (2009), 1075-1085 1

[8] A. Bak, R. Hazrat, N. Vavilov, Structure of hyperbolic unitary groups II. Normal subgroups, To appear. 4

[9] A. Bak, N. Vavilov, Normality for elementary subgroup functors, Math. Proc. Cambridge Philos. Soc. 118 (1995), no. 1, 35-47. 2, 4

[10] A. Bak, N. Vavilov, Structure of hyperbolic unitary groups I. Elementary subgroups, Algebra Colloq. 7 (2000), no. 2, 159-196. 2, 3, 4, 9

[11] A. Bak, N. Vavilov, Cubic form parameters, preprint, 2001. 3

[12] H. Bass, Unitary algebraic K-theory, Lecture Notes Math. 343 (1973), 57-265. 4

[13] Z. I. Borewicz, N. A. Vavilov, The distribution of subgroups in the full linear group over a commutative ring, Proc. Steklov Institute Math. 3 (1985), 27-46. 4

[14] R. W. Carter, Simple groups of Lie type, Wiley, London et al. 1972. 8

[15] D. L. Costa, G. E. Keller, Radix redux: normal subgroups of symplectic groups, J. reine angew. Math. 427 (1992), 51-105. 2, 6

[16] D. L. Costa, G. E. Keller, On the normal subgroups of $G(\Phi, R)_{2}(A)$, Trans. Amer. Math. Soc. 351 (1999), no.12, 5051-5088. 2, 6

[17] A. J. Hahn, O. T. O'Meara, The Classical Groups and K-Theory, Springer, Berlin 1989. 4

[18] R. Hazrat, Dimension theory and nonstable $K_{1}$ of quadratic modules, K-Theory 27 (2002), no. 4, 293-328. 4

[19] R. Hazrat, N. Vavilov, $K_{1}$ of Chevalley groups are nilpotent, J. of Pure and Appl. Algebra 179 (2003), 99-116. 2, 4

[20] R. Hazrat, N. Vavilov, Bak's work on the K-theory of rings J. K-Theory, 4 (2009), 1-65. 4

[21] J. F. Hurley, Ideals in Chevalley algebras, Trans. Amer. Math. Soc. 137 (1969), 245-258. 3, 4, 8

[22] J. F. Hurley, Some normal subgroups of elementary subgroups of Chevalley groups over rings, Amer. J. Math. 93 (1971), 1059-1069. 4 
[23] V. Kopeiko, The stabilisation of symplectic groups over polynomial rings, Math. USSR Sb. 34 (1978), 655-669. 4

[24] Li Fuan, The structure of symplectic group over arbitrary commutative rings, Acta Math. Sinica, New Series 3 (1987), no.3, 247-255. 4

[25] Li Fuan, The structure of orthogonal groups over arbitrary commutative rings, Chinese Ann. Math. 10 (1989), no.3, 341-350. 4

[26] H. Matsumoto, Sur les sous-groupes arithmétiques des groupes semi-simples déployés, Ann. Sci. École Norm. Sup. ser. 4, 2 (1969), 1-62. 3

[27] V. Petrov, Overgroups of unitary groups, K-Theory 29 (2003), no. 3, 147-174. 4

[28] V. A. Petrov, Odd unitary groups, J. Math. Sci. 130 (2003), no. 3, 4752-4766. 2, 4

[29] V. A. Petrov, A. K. Stavrova, Elementary subgroups of isotropic reductive groups, St. Petersburg Math. J. 20 (2008), no. 3 (Russian, English translation pending, see also PDMI preprint 1 (2008), 1-20). 4

[30] M. R. Stein, Chevalley groups over commutative rings, Bull. Amer. Math. Soc. 77 (1971), 247-252. 4

[31] M. R. Stein, Generators, relations and coverings of Chevalley groups over commutative rings, Amer. J. Math. 93 (1971), no.4, 965-1004. 3, 4, 5, 9

[32] M. R. Stein, Relativizing functors on rings and algebraic K-theory, J. Algebra 19 (1971), no.1, 140-152. 2

[33] A. V. Stepanov, On the normal structure of the general linear group over a ring, J. Math. Sci. 95 (1999), no.2, 2146-2155. 3, 4

[34] A. Stepanov, N. Vavilov, Decomposition of transvections: a theme with variations, K-Theory 19 (2000), 109-153. 4

[35] A. V. Stepanov, N. A. Vavilov, Standard commutator formulae, Vestnik Saint-Petersburg State Univ., ser.1 (2008), no.1, 9-14 (Russian, English translation pending). 4

[36] A. A. Suslin, On the structure of the general linear group over polynomial rings, Soviet Math. Izv. 41 (1977), no. 2 503-516. 4

[37] A. A. Suslin, V. I. Kopeiko, Quadratic modules and orthogonal groups over polynomial rings, J. Sov. Math. 20 (1985), no.6, 2665-2691. 4

[38] K. Suzuki, Normality of the elementary subgroups of twisted Chevalley groups over commutative rings, J. Algebra 175 (1995), no.3, 526-536. 2, 4

[39] G. Taddei, Normalité des groupes élémentaire dans les groupes de Chevalley sur un anneau, Contemp. Math. 55 II (1986), 693-710. 2, 4, 5

[40] L. N. Vaserstein, On the normal subgroups of $\mathrm{GL}_{n}$ over a ring, Lecture Notes Math. 854 (1981), 456-465. 4

[41] L. N. Vaserstein, On normal subgroups of Chevalley groups over commutative rings, Tôhoku Math. J. 36 (1986), no.5, 219-230. 1, 2, 4, 5

[42] N. Vavilov, Structure of Chevalley groups over commutative rings, Non-associative algebras and related topics, (Hiroshima, 1990), World Scientific Publishing, London et al., (1991), 219-335. 3, 4

[43] N. Vavilov, An $\mathrm{A}_{3}$-proof of structure theorems for Chevalley groups of types $\mathrm{E}_{6}$ and $\mathrm{E}_{7}$, Int. J. Algebra Comput. 17 (2007), no.5-6, 1283-1298. 4

[44] N. A. Vavilov, M. R. Gavrilovich, An $\mathrm{A}_{2}$-proof of structure theorems for Chevalley groups of types $\mathrm{E}_{6}$ and $\mathrm{E}_{7}$, St.-Petersburg Math. J. 16 (2005), no.4, 649-672. 4

[45] N. A. Vavilov, M. R. Gavrilovich, S. I. Nikolenko, Structure of Chevalley groups: the Proof from the Book, J. Math. Sci. 140 (2006), no.5, 626-645. 4

[46] N. A. Vavilov, S. I. Nikolenko, An $\mathrm{A}_{2}$-proof of structure theorems for Chevalley groups of type $\mathrm{F}_{4}$, St. Petersburg Math. J. 20 (2008), no. 3 (Russian, English translation pending). 2, 4, 6

[47] N. A. Vavilov, E. B. Plotkin, Net subgroups of Chevalley groups, J. Sov. Math. 19 (1982), no.1, 1000-1006. 2 
[48] N. A. Vavilov, E. B. Plotkin, Chevalley groups over commutative rings. I. Elementary calculations, Acta Appl. Math. 45 (1996), 73-115. 3, 8

[49] N. A. Vavilov, A. K. Stavrova, Basic reductions in the description of normal subgroups, J. Math. Sci. 349 (2007), 30-52 (Russian, English translation pending). 4, 6

Department of Pure Mathematics, Queen's University, Belfast BT7 1NN, U.K.

E-mail address: r.hazrat@qub.ac.uk

Department of Mathematics and Mechanics, St. Petersburg State University, St. PeTERSBURG 198504, RUSSIA

E-mail address: victorapetrov@gmail.com

Department of Mathematics and Mechanics, St. Petersburg State University, St. PeTERSBURG 198504, RUSSIA

E-mail address: nikolai-vavilov@yandex.ru 